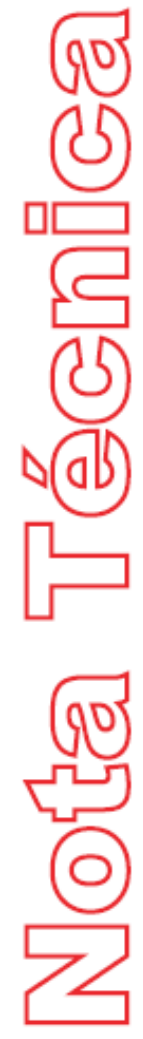

\title{
CARTOGRAFIA PRELIMINAR DOS FLUXOS E DESLOCAMENTOS DOS TERREIROS DE RELIGIOES DE MATRIZ AFRICANA E AFROBRASILEIRA DO DISTRITO FEDERAL PARA OUTRAS UNIDADES POLIITICAS DO BRASIL
}

\author{
Rafael Sanzio Araújo dos \\ Anjos Richard Denis \\ Raphael Parizotto \\ Estephany Almeida \\ Cleison Ferreira \\ Gustavo Tolentino
}

p. $31-37$

Revista

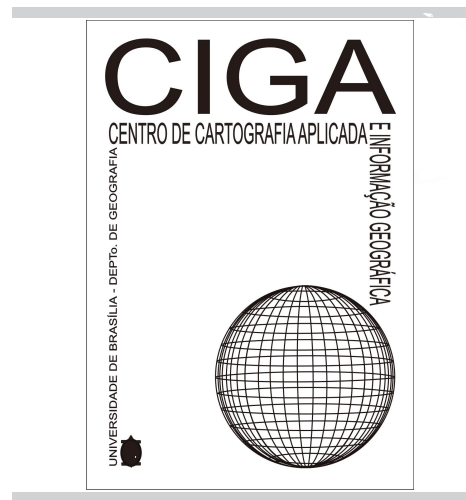

Revista Eletrônica:

Tempo - Técnica - Território, V.9, N.3 (2018), 31:37

ISSN : 2177-4366
Como citar este ar tigo:

Anjos, R.S.A; Denis, R.; Parizotto, R.; Almeida, E.; Ferreira, C.; Tolentino, G.; CARTOGRAFIA PRELIMINAR DOS FLUXOS E DESLOCAMENTOS DOS TERREIROS DE RELIGIÕES DE MATRIZ AFRICANA E AFROBRASILEIRA DO DISTRITO FEDERAL PARA OUTRAS UNIDADES POLÍTICAS DO BRASIL. Revista Eletrônica: Tempo - Técnica - Território, v.9, n.3 (2018), p. 31:37 ISSN: 2177-4366.

Disponível em:

http://periodicos.unb.br/index.php/ciga/article/view/17195/21288

Este obra está licenciado com uma Licença Creative Commons Atribuição-NãoComercial 4.0 Internacional. 


\title{
CARTOGRAFIA PRELIMINAR DOS FLUXOS E DESLOCAMENTOS DOS TERREIROS DE RELIGIÕES DE MATRIZ AFRICANA E AFROBRASILEIRA DO DISTRITO FEDERAL PARA OUTRAS UNIDADES POLÍTICAS DO BRASIL
}

\section{NOTA TÉCNICA}

\author{
Rafael Sanzio Araújo dos Anjos (*), Raphael Parizotto, Estephany Almeida, \\ Cleison Ferreira(**), Richard Denis, Gustavo Tolentino (***) \\ E-mail:cartografia@unb.br Site:www.ciga.unb.br
}

(*) Geógrafo, Doutor em Informações Espaciais Pós-Doutorado Cartografia Étnica. Prof. Titular da Universidade de Brasília / Diretor do Centro de Cartografia Aplicada e Informação Geográfica do Depto. de Geografia - UnB. Coordenador do Projeto do Mapeamento dos Terreiros do DF (Parceria Projeto GEOAFRO-CIGA-UnB-FinatecFCP-MINc (**)Geógrafos Membros da Equipe Técnica do Projeto do Mapeamento dos

Terreiros do DF (Parceria Projeto GEOAFRO-CIGA-UnB-Finatec-FCP$\operatorname{MINc}(* * *)$ Estagiários membros da Equipe Técnica do Projeto do Mapeamento dos Terreiros do DF (Parceria Projeto GEOAFRO-CIGA-UnB-Finatec-FCP-MINc)

RESUMO: A partir das investigações de campo do Projeto de Pesquisa do Mapeamento dos Terreiros do DF, realizado (2017-2018) no bojo da parceria institucional do Projeto GEOAFRO, do Centro de Cartografia Aplicada da Universidade de Brasília, da Fundação Cultural Palmares e da Finatec, foi possível verificar uma mobilidade espacial dos terreiros para municípios da Região do Entrono do Df e para outras unidades políticas do país. Esta Nota Técnica trata da cartografia elaborada para representar esta dinâmica geográfica relevante para as políticas públicas reparatórias. O estudo preliminar aponta para a necessidade de um estudo mais apurado para contabilizar as origem-destinos e o conjunto complexo de motivos que estão levando os Terreiros Religiosos de Mtariz Africana no DF a irem embora.

ABSTRACT : Based on the field investigations of the Terreiros Mapping Research Project in the Federal District (2017-2018), in the context of the GEOAFRO Project's institutional partnership, the Center for Applied Cartography of the University of Brasília, the Palmares Cultural Foundation and Finatec, it was possible to verify a spatial mobility of the terreiros to municipalities of the Entrono Region of the Federal District and to other political units of the country. This Technical Note deals with cartography designed to represent this geographic dynamic relevant to public reparatory policies. The preliminary study points to the need for a more accurate study to account for the origin-destinations and the complex set of motives that are driving the African Religious Mtariz Terreiros in the DF to leave. 


\section{A CARTOGRAFIA, O ORDENAMENTO DO TERRITÓRIO}

O conhecimento acerca das informações espaciais constitui relevante instrumento para a gestão e planejamento do território, competindo dessa forma, ao geografo, a leitura dos elementos espaciais e sua interpretação de forma clara e objetiva.Nesse sentido, as informações geográficas se caracterizam como elementos estratégicos, de modo que a partir dela possibilite responder as diferentes formas e expressões existentes no espaço.

Tendo como ponto de referencia a pesquisa de Mapeamento dos Terreiros de Umbanda e Candomblé do Distrito Federal do Brasil, $1^{\text {a }}$ Etapa - Cartografia Básica (2018), será apresentado os pressupostos instrumentais e metodológicos adotados na produção cartográfica do Mapa de Fluxo dos Terreiros de Umbanda e Candomblé do Distrito Federal para os outros Estados do Território Brasileiro. Com essa produção, busca-se ampliar a contribuição sobre o conhecimento cartográfico, do espaço e suas manifestações no enfoque da pesquisa realizada.

\section{O CIGA}

O Centro de Cartografia Aplicada e Informação Geográfica (CIGA) constitui um importante espaço para o desenvolvimento de atividades, projetos e parcerias dentro da Universidade de Brasília (UnB), oferecendo uma estrutura organizada e equipamentos básicos para o desenvolvimento de estudos relacionados aosdiversos campos de atuação geográfica. O CIGA atua em duas linhas de pesquisa fundamentais, a primeira, a Geografia Afro-Brasileira, e seus segmentos, a segunda, a Instrumentação Geográfica, Educação e Planejamento dos Territórios com seus segmentos. Nesse âmbito, o CIGA, aliado com a cartografia, possibilita tratar e produzir estudos que nos ajudam a compreender as dinâmicas passadas, atuais e futuras atuantes o espaço geográfico.

\section{O MAPA DOS FLUXOS DOS TERREIROS DE UMBANDA E CANDOMBLÉ DO DISTRITO FEDERAL PARA OS OUTROS ESTADOS DOS TERRITÓRIOS BRASILEIRO - 2018}

Com o desenvolvimento da pesquisa de Mapeamento dos Terreiros de Umbanda e Candomblé do Distrito Federal do Brasil, $1^{\mathrm{a}}$ Etapa - Cartografia Básica (2018),surgiram alguns questionamentos quanto à quantidade de terreiros de matrizes africanas situados no território do Distrito Federal, existindo inicialmente uma 
estimativa de aproximadamente 300 terreiros.Porém, com os andamentos dos trabalhos de campo, coleta das informações geográficas e elaboração do banco de dados, percebeu-se notável fluxo migratório dos terreiros para outras localidades, esse movimento, muitas vezes em direção a cidades integrantes da Região Integrada de Desenvolvimento Econômico (RIDE) e até mesmo para outros estados da federação brasileira.

Devido à relevância do assunto, fora produzido o Mapa dos Fluxos dos Terreiros de Umbanda e Candomblé do Distrito Federal para outros Estados do Território Brasileiro, com a finalidade de reproduzir cartograficamente os novos fluxos e destinos dos terreiros. Para esse fim, foi utilizada a base cartográfica da América do Sul e do Brasil (CIGA, 2015), os registros de mudanças para fora do DF, apoiados na base de dados do projeto, e consultorias com representantes da Fundação Cultural Palmares (FCP). As figuras 1 e 2 mostram elementos do processo metodológico de produção do mapa.

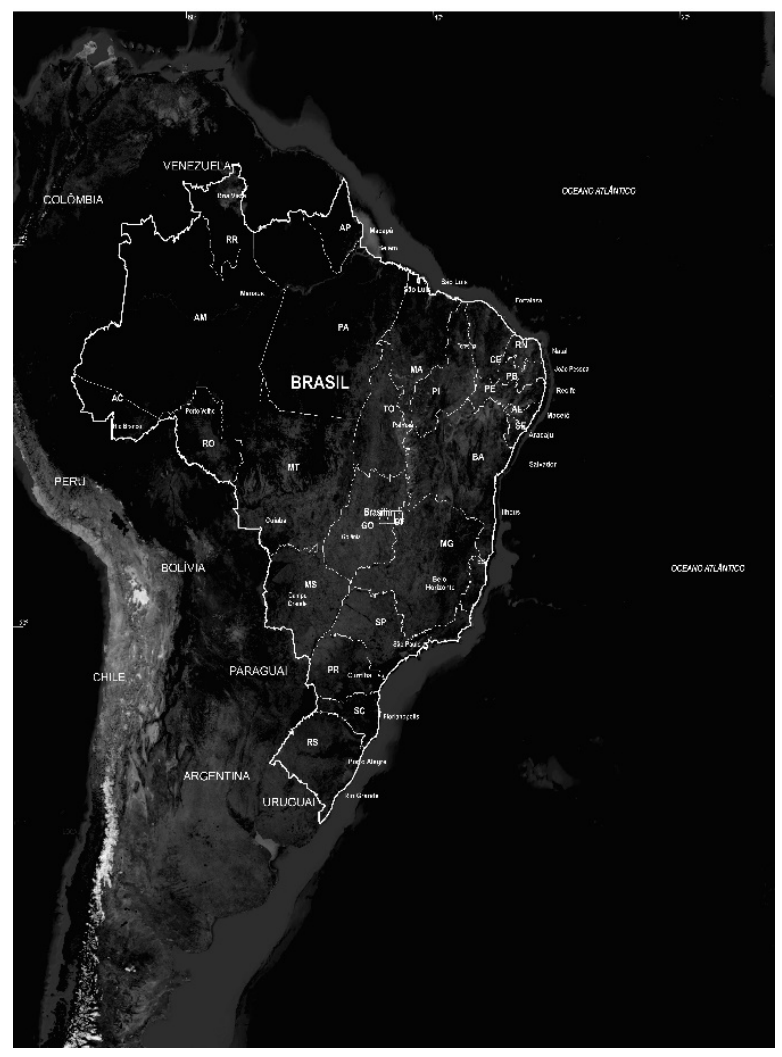

\begin{tabular}{|c|c|c|}
\hline \multicolumn{3}{|c|}{ FLUXO EXTERNO MAPA RIDE E BRASIL } \\
\hline LOCAL & ORIGEM & DESTINO \\
\hline RA010005 & BRASÍLIA & LUZIÂNIA/GO \\
\hline RA010015 & BRASÍLIA & $\begin{array}{l}\text { STO.ANTÔNIO } \\
\text { DESC./GO }\end{array}$ \\
\hline RA020003 & GAMA & GOIÁS/GO \\
\hline RA030007 & TAGUATINGA & ÁGUAS LINDAS/GO \\
\hline RA030015 & TAGUATINGA & GOIÁS/GO \\
\hline RA030019 & TAGUATINGA & GOIÁS/GO \\
\hline RA030024 & TAGUATINGA & OCIDENTAL/GO \\
\hline RA060015 & PLANALTINA & PLANALTINA/GO \\
\hline RA060025 & PLANALTINA & RIO DE JANEIRO/RJ \\
\hline RA090021 & CEILÂNDIA & GOIÁS/GO \\
\hline RA120011 & SAMAMBAIA & PIAUI/PI \\
\hline RA130006 & SANTA MARIA & CEARÁ/CE \\
\hline RA130011 & SANTA MARIA & OCIDENTAL/GO \\
\hline RA180006 & LAGO NORTE & LUZIÂNIA/GO \\
\hline RA210003 & $\begin{array}{l}\text { RIACHO FUNDO } \\
\text { II }\end{array}$ & GOIÁS/GO \\
\hline & $\begin{array}{l}\text { DISTRITO } \\
\text { FEDERAL }\end{array}$ & SÃO PAULO E BAHIA \\
\hline
\end{tabular}

Figura 1. Base cartográfica para o mapa. Figura 2. Tabela da base de dados. 
Para a manipulação das informações geográficas, foram utilizados os softwares como o CorelDraw e o Microsoft Office Excel, programas que possibilitaram a localização dos pontos, criação dos desenhos e elementos gráficos e organização dos dados em planilhas. A figura 3 expressa a tela de trabalho no CorelDraw.

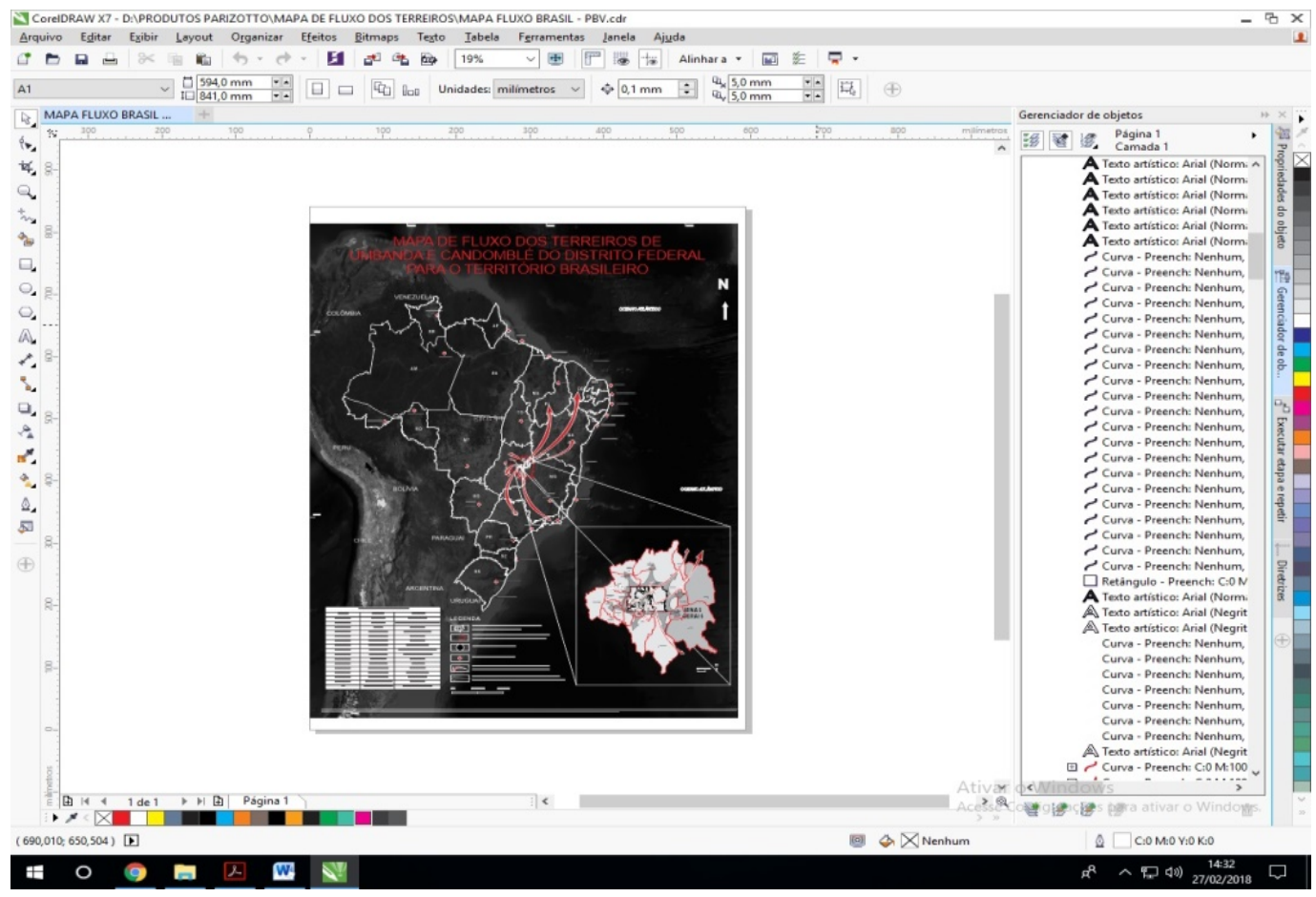

Figura 3. Tela de trabalho no software CorelDraw.

\section{PRODUÇÕES CARTOGRÁFICAS}

Como base para a confecção do mapa, foi utilizada a base cartográfica da América do Sul e do Brasil (CIGA, 2015), compreendendo a região da América do Sul e os Oceanos Atlântico e Pacífico. Utilizando o software CorelDraw fora elaborados os limites geopolíticos do território brasileiro e seus respectivos estados federados.

Na Figura 4 podem ser observadas as diferenças da imagem original para a trabalhada, onde, se tornam perceptíveis com a inclusão dos limites territoriais e inclusão de elementos cartográficos. 

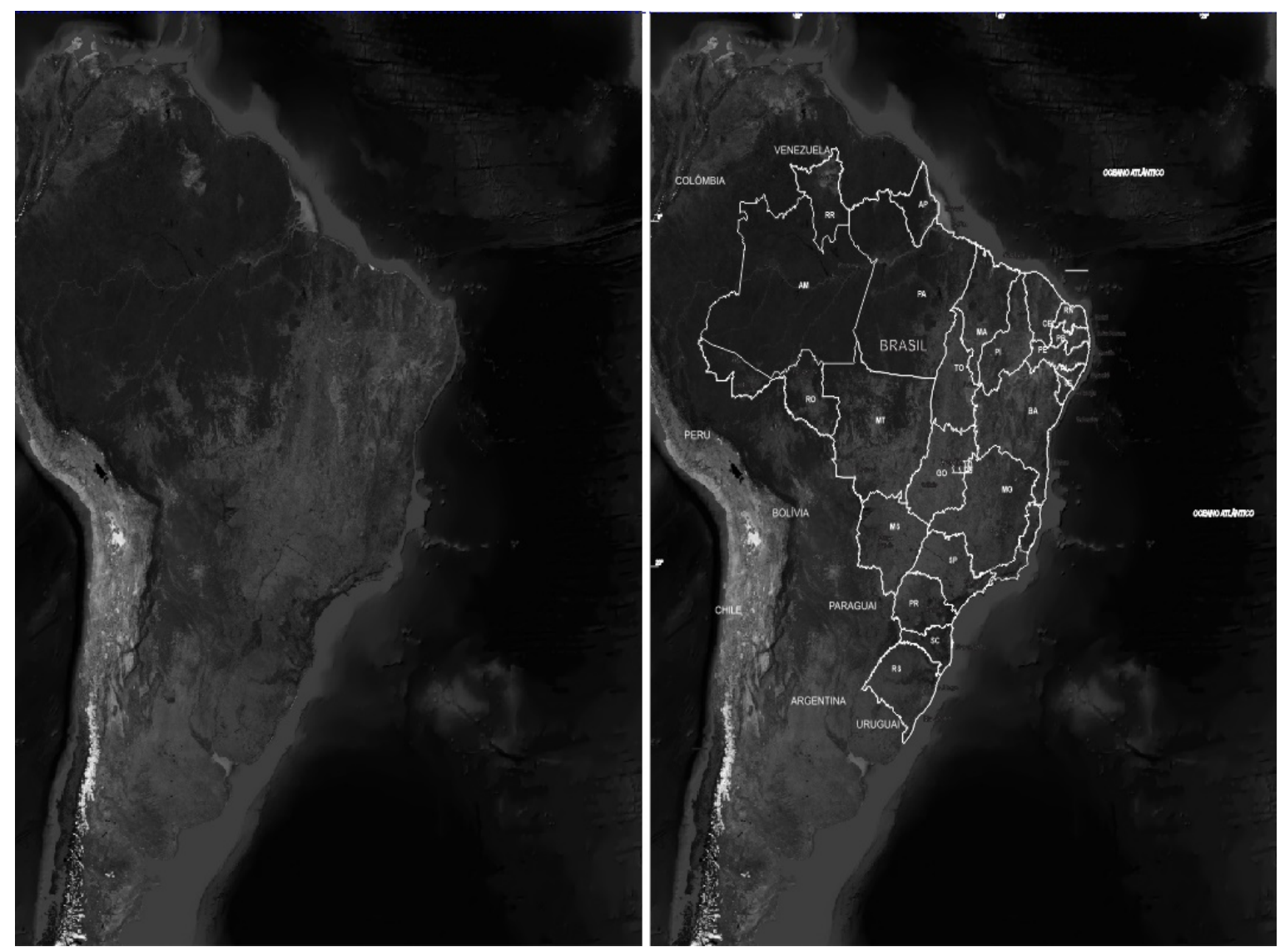

Figura 4. Produção em cima da base cartográfica da América do Sul, foco no Brasil.

A etapa seguinte se caracterizou com as representações dos fluxos migratórios dos terreiros, as representações seguiram duas formas distintas, a primeira, com base nas informações ponto a ponto, ou seja, dos terreiros que se tem conhecimento do ponto de origem até o seu ponto de destino, esses casos foram representados por uma seta vermelha no mapa. O segundo caso, diverge do primeiro quanto à precisão da informação, sendo sabido apenas o deslocamento do terreiro para outra região do estado de Goiás, para representar esses fluxos, fora utilizado uma seta em forma de mancha, permitindo uma ideia de avanços para o interior do estado.

\section{ELEMENTOS DA LEGENDA}

Para o mapa de fluxos dos terreiros, em sua legenda são representados itens relevantes para a compreensão e interpretação das informações, entre elas: as setas de fluxo dos terreiros com origem e destino conhecidos; as setas em forma de mancha dos terreiros com origem e destino para o estado do Goiás (cidade incerta); limite político 
do atual território brasileiro; limite em vermelho da área que abrange a RIDE-DF; elipse na cor preta, simbolizando a capital federal do Brasil; elipse na cor preta, simbolizando as demais capitais estaduais do Brasil.

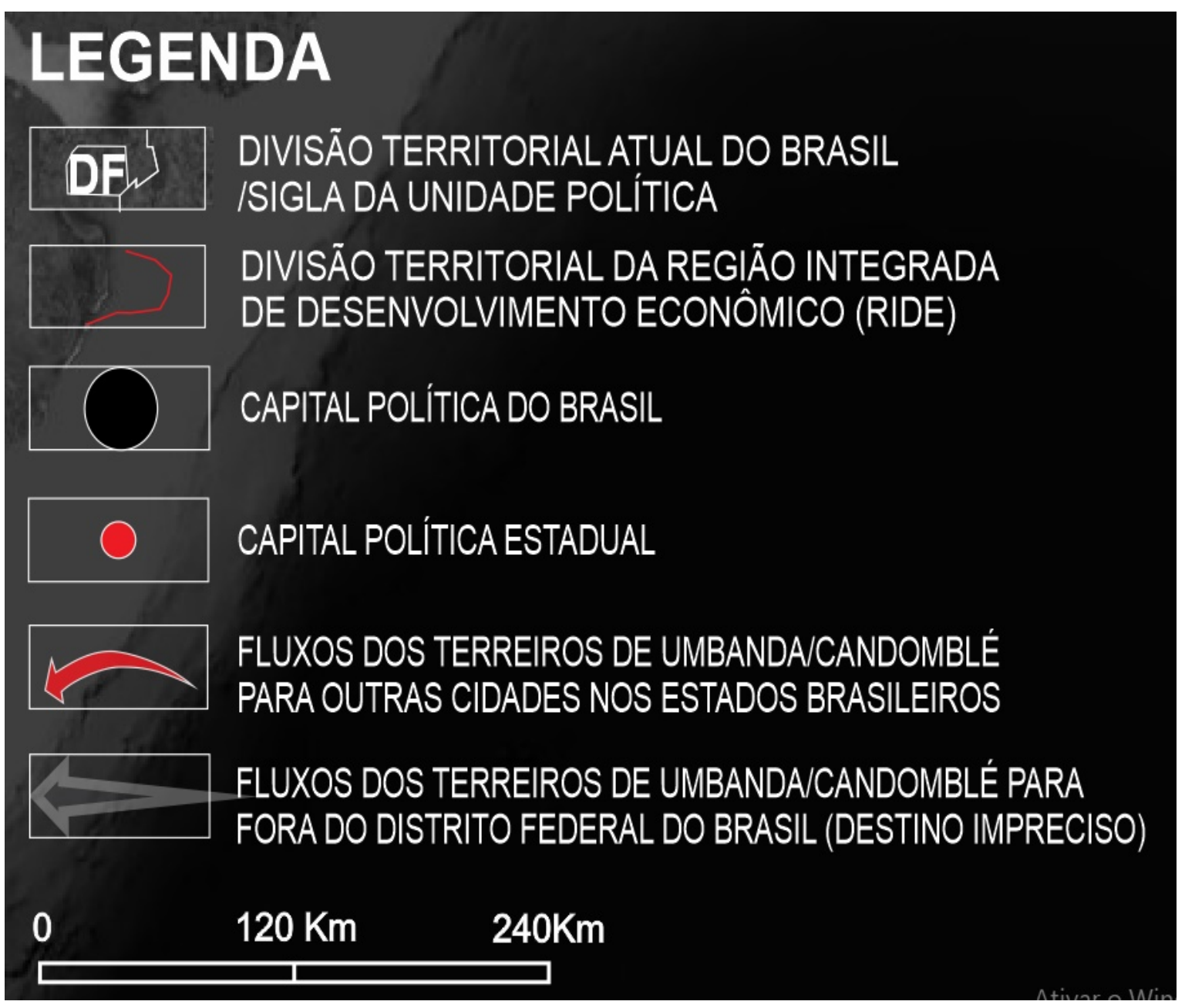

Figura 5. Categorias de legenda adotadas no mapa.

\section{PRODUTO FINAL}

Como produto final, fora realizado duas produções do mesmo mapa, uma com diferentes paletas de cores e outro com as cores: preta vermelha e tons de cinza. Seguem abaixo as figuras dos produtos finalizados. 


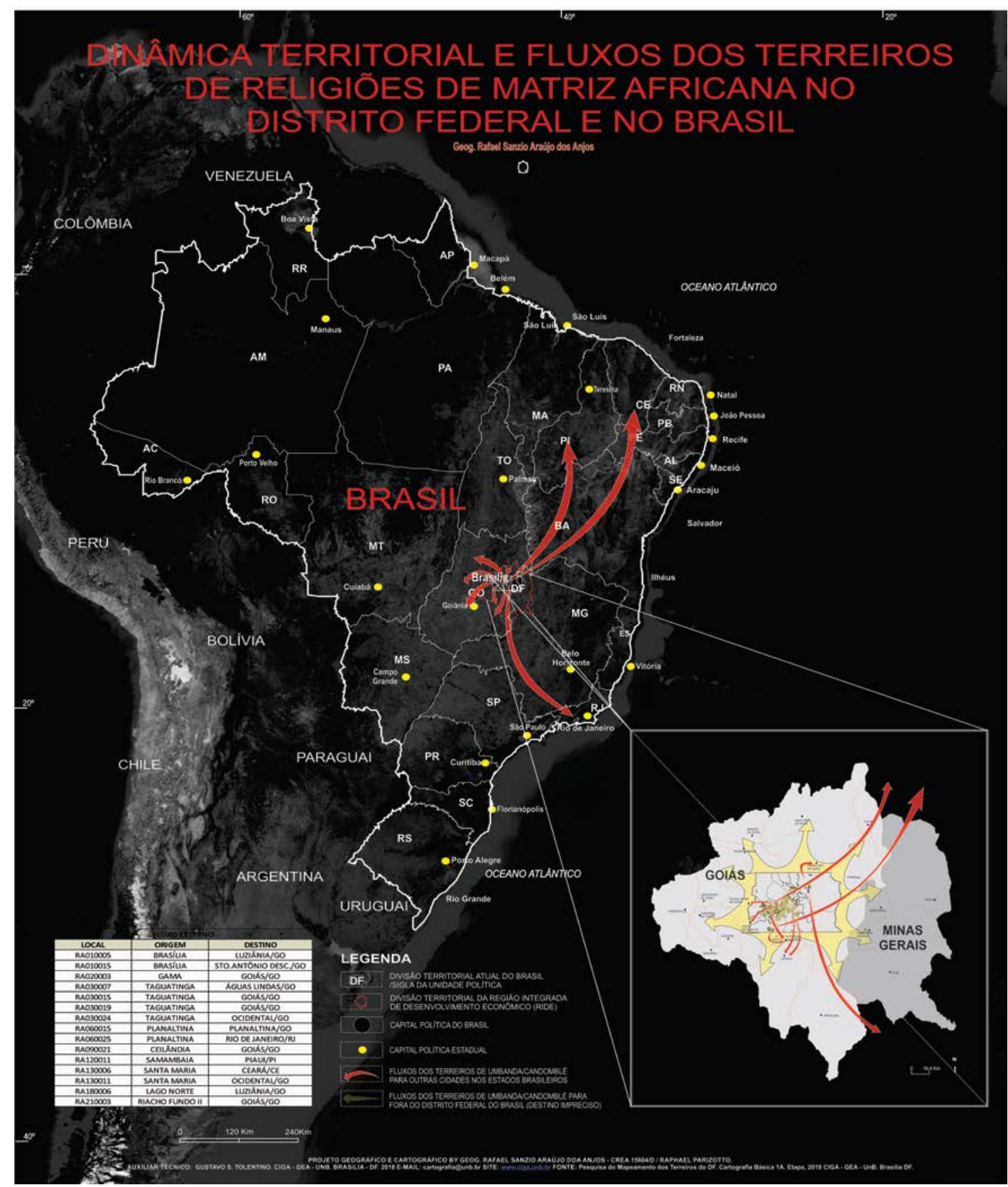

Figura 6. Versão final com diferentes paletas de cores do Mapa de Fluxo dos Terreiros de Umbanda e Candomblé do Distrito Federal para o Território Brasileiro.

\section{METATADO ESPACIAL}

Para o mapa de fluxos dos terreiros, em sua legenda são representados itens relevantes

\section{BIBLIOGRAFIA}

ANJOS, R.S.A. Mapeamento dos terreiros do Distrito Federal: Cartografia básica 1A. Etapa. Instituto Baobás, Projeto GEOAFRO, CIGA-UnB , Coord. Brasília, DF. 2018

Revista Eletrônica: Tempo - Técnica - Território, V.9, N.3 (2018), 31:37 ISSN: 2177-4366 